\title{
Intrinsic Localization of Anisotropic Frames II: $\alpha$-Molecules
}

\author{
Philipp Grohs and Stefano Vigogna*
}

\begin{abstract}
This article is a continuation of the recent paper 21 by the first author, where off-diagonal-decay properties (often referred to as 'localization' in the literature) of Moore-Penrose pseudoinverses of (bi-infinite) matrices are established, whenever the latter possess similar off-diagonal-decay properties. This problem is especially interesting if the matrix arises as a discretization of an operator with respect to a frame or basis. Previous work on this problem has been restricted to wavelet- or Gabor frames. In 21. we extended these results to frames of parabolic molecules, including curvelets or shearlets as special cases. The present paper extends and unifies these results by establishing analogous properties for frames of $\alpha$-molecules as introduced in recent work 22. Since wavelets, curvelets, shearlets, ridgelets and hybrid shearlets all constitute instances of $\alpha$-molecules, our results establish localization properties for all these systems simultaneously.
\end{abstract}

Keywords: Frame Localization, Curvelets, Shearlets, Ridgelets, Wavelets, nonlinear Approximation. AMS Classifiers: Primary 41AXX, Secondary 41A25, 53B, 22E.

\section{Introduction}

This article, which is continuation of our earlier work 21, studies off-diagonal decay properties of MoorePenrose pseudoinverses $A^{+}$of symmetric (bi-infinite) matrices $A=\left(A_{\lambda, \lambda^{\prime}}\right)_{\lambda, \lambda^{\prime} \in \Lambda}$, with $\Lambda$ a discrete index set. More precisely, our results are of the following general type: assume that $A$ is localized, in the sense that

$$
\left|A_{\lambda, \lambda^{\prime}}\right| \leq C \omega\left(\lambda, \lambda^{\prime}\right)^{-N} \text { for all } \lambda, \lambda^{\prime} \in \Lambda
$$

with respect to some nice function $\omega$ measuring the distance between the indices. Then the Moore-Penrose pseudoinverse of $A$ satisfies the analogous inequality with a different constant $C$ and a parameter $N^{+} \leq N$ which we describe explicitly.

Typically $A$ arises as a Gram matrix $A=\left(\left\langle\psi_{\lambda}, \psi_{\lambda^{\prime}}\right\rangle_{\mathcal{H}}\right)_{\lambda, \lambda^{\prime} \in \Lambda}$ of a frame $\left(\psi_{\lambda}\right)_{\lambda \in \Lambda}$ of a Hilbert space $\mathcal{H}$. In that case the Moore-Penrose pseudoinverse $A^{+}$corresponds to the Gram matrix of the canonical dual frame of $\left(\psi_{\lambda}\right)_{\lambda \in \Lambda}$. Hence, localization properties of $A^{+}$provides useful information about the canonical dual frame. For more information regarding frames we refer to 11. For a more detailed motivation of the problem that we consider in the present paper (for instance in the context of operator compression) we refer to our earlier work [21].

The 'localization problem' as described above has been studied in several contexts, see 1, 3, 4, 2, 7, 5, 6, 18, 15, 12, 30, 31, 13, 26, 17, 28. In these works the index set $\Lambda$ arises as a sampling set for either Gaboror wavelet frames. In both cases there exists a canonical index distance function $\omega$ for which localization results have been established in the aforementioned works. Recently, these results have been extended to anisotropic frame systems such as curvelets [10] or shearlets [29, and more generally parabolic molecules 23.

The present paper extends and unifies these results. More precisely, we shall prove localization results for index distance functions $\omega$ which are associated with frames of so-called $\alpha$-molecules as introduced in 22. The notion of $\alpha$-molecules includes wavelets, ridgelets 9, 19, shearlets, curvelets, parabolic molecules and $\alpha$-shearlets 27] as special cases. Consequently, the results of the present paper are applicable to all these systems at once.

Outline. We proceed as follows. In Section 2 we provide an abstract framework for index distance functions in which localization results can be established. The main result of this section is Theorem (2.14) which states that, if an index distance function $\omega$ satisfies certain properties, then localization of a matrix

* This work has been carried out during a 2 month visit of the second named author at ETH Zürich in 2013. He would like to take this opportunity to thank ETH Zürich for its hospitality and financial support. 
$A$ in the sense of (1.1) implies a similar property for its Moore-Penrose pseudoinverse $A^{+}$. To further motivate the importance of localization properties we also provide several results stating that localized matrices are automatically bounded on a wide class of weighted $\ell^{p}$ Banach spaces.

Then in Section 3 we apply the abstract framework of Section 2 to specific index distance functions, namely those associated with frames of $\alpha$-molecules as introduced in 22. More precisely, we verify that those index distance functions satisfy the assumptions of the abstract theory developed in Section 2 and hence provide localization results for the whole class of $\alpha$-molecules.

We collect some auxiliary results in Appendix A

\section{Abstract Framework}

In the present section we set the abstract framework which we later apply in Section 3 to establish localization results for frames of $\alpha$-molecules.

Subsection 2.1 below starts by introducing the kind of index distance functions $\omega$ with which we are working. We consequently define the Banach space of localized matrices, for which a submultiplicativity property is established in Theorem (2.7). This property provides a key technical tool to prove the main result of the section, namely Theorem (2.14). In Subsection 2.2 we show that localization with respect to such index functions implies boundedness on a large range of weighted $\ell^{p}$ spaces. Finally, in Subsection 2.3 we establish Theorem (2.14), which states the localization of the Moore-Penrose pseudoinverse of matrices which are localized with respect to $\omega$ as introduced in Subsection 2.1. Most of the material in this section is well-known. Using the proof techniques developed in 21, Theorem (2.14) is not too hard to establish. The difficult part of the present paper is contained in Section 3 , where we shall verify that canonical index distances associated to $\alpha$-molecules fit into the abstract framework developed in the present section.

\subsection{Basic Notions}

We shall prove a localization result in a general framework which we describe in the present section. Here we introduce the notations and definitions which we shall use, starting with the following definition of an index distance function.

(2.1) Definition. Let $\Lambda$ be a discrete index set. An index distance is a function $\omega: \Lambda \times \Lambda \longrightarrow[1, \infty)$ such that there exist constants $C_{S}, C_{T} \geq 1$ with

(i) $\omega(\lambda, \lambda)=1$ for all $\lambda \in \Lambda$;

(ii) $\omega\left(\lambda, \lambda^{\prime}\right) \leq C_{S} \omega\left(\lambda^{\prime}, \lambda\right)$ for all $\lambda, \lambda^{\prime} \in \Lambda$;

(iii) $\omega\left(\lambda, \lambda^{\prime}\right) \leq C_{T} \omega\left(\lambda, \lambda^{\prime \prime}\right) \omega\left(\lambda^{\prime \prime}, \lambda^{\prime}\right)$ for all $\lambda, \lambda^{\prime}, \lambda^{\prime \prime} \in \Lambda$.

(2.2) Definition. We say that $\Lambda$ is separated by $\omega$ if

$C_{\Lambda}:=\inf _{\lambda \neq \lambda^{\prime}} \omega\left(\lambda, \lambda^{\prime}\right)>1$

(2.3) Definition. Let $K \geq 1$. We say that $\omega$ is $K$-admissible if

$C_{\omega}:=\sup _{\lambda \in \Lambda} \sum_{\lambda^{\prime} \in \Lambda} \omega\left(\lambda, \lambda^{\prime}\right)^{-K}<\infty$.

(2.4) Remark. The pseudo-symmetry property (2.1) (iil) is not strictly necessary. However, our examples of index distance are all pseudo-symmetric in a natural way, and this allows to state the Schur type condition (2.3) in any fixed order of the indices. Furthermore, one can always replace $\omega\left(\lambda, \lambda^{\prime}\right)$ with its symmetrization $\omega^{\text {sym }}\left(\lambda, \lambda^{\prime}\right):=\frac{1}{2}\left(\omega\left(\lambda, \lambda^{\prime}\right)+\omega\left(\lambda^{\prime}, \lambda\right)\right)$ : if $\omega$ enjoys (2.1)(1) and (iii), (2.2) and (2.3) with constants $C_{T}, C_{\Lambda}$ and $C_{\omega}$, then $\omega^{\mathrm{sym}}$ will enjoy the same properties with constants $2 C_{T}$, inf $\lambda_{\lambda \neq \lambda^{\prime}} \omega^{\mathrm{sym}}\left(\lambda, \lambda^{\prime}\right) \geq C_{\Lambda}$ and $\sup _{\lambda \in \Lambda} \sum_{\lambda^{\prime} \in \Lambda} \omega^{\operatorname{sym}}\left(\lambda, \lambda^{\prime}\right)^{-K}<2^{K} C_{\omega}$.

On the contrary, the pseudo-triangle inequality (2.1) iiil is technically crucial.

Having introduced the required properties of an index distance function we now define the Banach space of localized operators. 
(2.5) Definition. Let $\omega$ be an admissible index distance and $N \geq 1$. A matrix $A \in \mathbb{C}^{\Lambda \times \Lambda}$ is said to be $N$-localized (with respect to $\omega$ ) if $\left|A_{\lambda, \lambda^{\prime}}\right| \lesssim \omega\left(\lambda, \lambda^{\prime}\right)^{-N}$ for all $\lambda, \lambda^{\prime} \in \Lambda$. We define $\mathcal{B}_{N}$ as the space of all $N$-localized matrices,

$$
\mathcal{B}_{N}:=\left\{A \in \mathbb{C}^{\Lambda \times \Lambda}:\left|A_{\lambda, \lambda^{\prime}}\right| \lesssim \omega\left(\lambda, \lambda^{\prime}\right)^{-N} \text { for all } \lambda, \lambda^{\prime} \in \Lambda\right\}
$$

with associated norm

$$
\|A\|_{\mathcal{B}_{N}}:=\inf \left\{C>0:\left|A_{\lambda, \lambda^{\prime}}\right| \leq C \omega\left(\lambda, \lambda^{\prime}\right)^{-N} \text { for all } \lambda, \lambda^{\prime} \in \Lambda\right\}=\sup _{\lambda, \lambda^{\prime} \in \Lambda} \omega\left(\lambda, \lambda^{\prime}\right)^{N}\left|A_{\lambda, \lambda^{\prime}}\right| .
$$

Notice that $\mathcal{B}_{N} \subseteq \mathcal{B}_{M}$ as $N \geq M$. We next show that $\mathcal{B}_{N}$ is complete.

(2.6) Proposition. The set $\mathcal{B}_{N}$ constitutes a Banach space with respect to the norm \|\|$_{\mathcal{B}_{N}}$.

Proof. Take a Cauchy sequence $\left(A_{n}\right)$ in $\mathcal{B}_{N}$. This means that $\omega^{N}\left(A_{n}\right)$ is uniformly Cauchy. Moreover, $\left(A_{n}\right)$ is pointwise Cauchy, since

$$
\left|A_{n}\left(\lambda, \lambda^{\prime}\right)-A_{m}\left(\lambda, \lambda^{\prime}\right)\right| \leq\left\|A_{n}-A_{m}\right\|_{\mathcal{B}_{N}} \omega\left(\lambda, \lambda^{\prime}\right)^{-N}
$$

Hence $\left(A_{n}\right)$ converges pointwise to some $A \in \mathbb{C}^{\Lambda}$. Now $\omega^{N}\left(A_{n}\right)$ converges pointwise to $\omega^{N} A$, and it is uniformly Cauchy, therefore it converges uniformly to $\omega^{N} A$. Then, since $\sup \omega^{N} A_{n}<\infty$ for all $n$, we also have $\sup \omega^{N} A<\infty$, namely $A \in \mathcal{B}_{N}$.

We close this subsection with the following result regarding the action of $\mathcal{B}_{M}$ on $\mathcal{B}_{N}$, whenever $M$ is sufficiently large, made possible by a submultiplicativity property of the $\mathcal{B}_{N}$-norm. This result is crucial for the proof of our main Theorem (2.14) Notice that, unlike [21, Proposition 2.13], it is not required that $C_{S}=1$ or $A B$ be symmetric.

(2.7) Theorem. Let $\Lambda$ be a discrete set, separated by a $K$-admissible index distance $\omega$. Let $A \in \mathcal{B}_{N+L}$ with $L \geq \max \left(2 N \log _{C_{\Lambda}} C_{T}, 2 K\right)$, and $B \in \mathcal{B}_{N}$. Then $A B \in \mathcal{B}_{N}$, with

$$
\|A B\|_{\mathcal{B}_{N}} \leq\left(1+C_{\omega}\right)\|A\|_{\mathcal{B}_{N+L}}\|B\|_{\mathcal{B}_{N}} .
$$

Proof. We have

$$
\begin{aligned}
& \left|(A B)_{\lambda, \lambda^{\prime}}\right|=\left|\sum_{\lambda^{\prime \prime} \in \Lambda} A_{\lambda, \lambda^{\prime \prime}} B_{\lambda^{\prime \prime}, \lambda^{\prime}}\right| \\
& \lesssim \sum_{\lambda^{\prime \prime} \in \Lambda} \omega\left(\lambda, \lambda^{\prime \prime}\right)^{-N-L} \omega\left(\lambda^{\prime \prime}, \lambda^{\prime}\right)^{-N} \quad \text { by }(2.5) \\
& =\omega(\lambda, \lambda)^{-N-L} \omega\left(\lambda, \lambda^{\prime}\right)^{-N}+\sum_{\lambda^{\prime \prime} \neq \lambda} \omega\left(\lambda, \lambda^{\prime \prime}\right)^{-N-L} \omega\left(\lambda^{\prime \prime}, \lambda^{\prime}\right)^{-N} \\
& =\omega\left(\lambda, \lambda^{\prime}\right)^{-N}+\sum_{\lambda^{\prime \prime} \neq \lambda} \omega\left(\lambda, \lambda^{\prime \prime}\right)^{-N-L} \omega\left(\lambda^{\prime \prime}, \lambda^{\prime}\right)^{-N} \text { by (2.1) (i) } \\
& =\omega\left(\lambda, \lambda^{\prime}\right)^{-N}+\sum_{\lambda^{\prime \prime} \neq \lambda}\left[\omega\left(\lambda, \lambda^{\prime \prime}\right) \omega\left(\lambda^{\prime \prime}, \lambda^{\prime}\right)\right]^{-N} \omega\left(\lambda, \lambda^{\prime \prime}\right)^{-L} \\
& \leq \omega\left(\lambda, \lambda^{\prime}\right)^{-N}+C_{T}{ }^{N} \omega\left(\lambda, \lambda^{\prime}\right)^{-N} \sum_{\lambda^{\prime \prime} \neq \lambda} \omega\left(\lambda, \lambda^{\prime \prime}\right)^{-L} \text { by (2.1) (iii) } \\
& =\omega\left(\lambda, \lambda^{\prime}\right)^{-N}\left(1+C_{T}^{N} \sum_{\lambda^{\prime \prime} \neq \lambda} \omega\left(\lambda, \lambda^{\prime \prime}\right)^{-L / 2} \omega\left(\lambda, \lambda^{\prime \prime}\right)^{-L / 2}\right) \\
& \leq \omega\left(\lambda, \lambda^{\prime}\right)^{-N}\left(1+C_{T}^{N} C_{\Lambda}^{-L / 2} \sum_{\lambda^{\prime \prime} \neq \lambda} \omega\left(\lambda, \lambda^{\prime \prime}\right)^{-L / 2}\right) \quad \text { by }(2.2) \\
& \leq \omega\left(\lambda, \lambda^{\prime}\right)^{-N}\left(1+\sum_{\lambda^{\prime \prime} \neq \lambda} \omega\left(\lambda, \lambda^{\prime \prime}\right)^{-L / 2}\right) \quad \text { as } L \geq 2 N \log _{C_{\Lambda}} C_{T} \\
& \leq \omega\left(\lambda, \lambda^{\prime}\right)^{-N}\left(1+C_{\omega}\right) \quad \text { as } L \geq 2 K, \text { by }(2.3) \text {. }
\end{aligned}
$$




\subsection{Localization implies Boundedness}

One important feature of localization is that it implies boundedness on a large class of weighted $\ell^{p}$ spaces. A classical instance of this type of results are boundedness results for Calderòn-Zygmund operators on Besov spaces, which can be shown by representing the operators in a wavelet basis and using localization, together with the fact that Besov space norms can be characterized in terms of weighted $\ell^{p}$ norms of wavelet coefficients 16. As another example we mention Fourier integral operators which can be shown to be localized if represented in a frame of parabolic molecules [21, 14. Consequently, such operators are bounded on the associated functions spaces, as described e.g. in [8].

In the present section we establish results stating that localized matrices always induce bounded operators on weighted $\ell^{p}$ spaces, whenever the index distance $\omega$ satisfies certain admissibility properties.

Given a weight function $\mathrm{w}: \Lambda \rightarrow(0, \infty)$, for $p \in(0, \infty]$ we define the weighted $\ell^{p}$ spaces

$$
\ell_{\mathrm{w}}^{p}(\Lambda):=\left\{a \in \mathbb{C}^{\Lambda}: a \mathrm{w} \in \ell^{p}(\Lambda)\right\}
$$

with weighted norms

$$
\|a\|_{p, \mathrm{w}}:=\|a \mathrm{w}\|_{p}
$$

where we write $a \mathrm{w}=(a(\lambda) \mathrm{w}(\lambda))_{\lambda \in \Lambda}$. We recall the following weighted version of the Schur test ([24, Lemma 4]).

(2.8) Lemma. Let $A \in \mathbb{C}^{\Lambda \times \Lambda}$. For $\mathrm{w}_{1}, \mathrm{w}_{2}: \Lambda \rightarrow(0,+\infty)$ and $p_{0} \in(0,1]$, consider the Schur conditions

$$
\begin{aligned}
& \sum_{\lambda \in \Lambda} \mathrm{w}_{2}(\lambda)^{p_{0}}\left|A_{\lambda, \lambda^{\prime}}\right|^{p_{0}} \leq C_{1}^{p_{0}} \mathrm{w}_{1}\left(\lambda^{\prime}\right)^{p_{0}} \text { for some } C_{1}>0, \\
& \sum_{\lambda^{\prime} \in \Lambda}\left|A_{\lambda, \lambda^{\prime}}\right| \mathrm{w}_{1}\left(\lambda^{\prime}\right)^{-1} \leq C_{2} \mathrm{w}_{2}(\lambda)^{-1} \text { for some } C_{2}>0,
\end{aligned}
$$

and define the formal matrix operator

$$
(A a)_{\lambda}:=\sum_{\lambda^{\prime} \in \Lambda} A_{\lambda, \lambda^{\prime}} a_{\lambda^{\prime}} \quad a \in \mathbb{C}^{\Lambda}
$$

Then:

(a) if $A$ enjoys (2.9)a, then it is bounded from $\ell_{\mathrm{w}_{1}}^{p}(\Lambda)$ to $\ell_{\mathrm{w}_{2}}^{p}(\Lambda)$ for all $p \in\left[p_{0}, 1\right]$;

(b) if $A$ enjoys (2.9)b, then it is bounded from $\ell_{\mathrm{w}_{1}}^{\infty}(\Lambda)$ to $\ell_{\mathrm{w}_{2}}^{\infty}(\Lambda)$;

(c) if $A$ enjoys (2.9)a and (2.9)b, then it is bounded from $\ell_{\mathrm{w}_{1}}^{p}(\Lambda)$ to $\ell_{\mathrm{w}_{2}}^{p}(\Lambda)$ for all $p \in\left[p_{0}, \infty\right]$.

In each case, $\|A\|_{\ell_{\mathrm{w}_{1}}^{p} \rightarrow \ell_{\mathrm{w}_{2}}^{p}} \leq C_{1}^{1 / p} C_{2}^{1 / p^{\prime}}$ for $p \in[1, \infty]$ (where $1 / p+1 / p^{\prime}=1$ and $\left.1 / \infty=0\right)$, and $\|A\|_{\ell_{\mathrm{w}_{1}}^{p} \rightarrow \ell_{\mathrm{w}_{2}}^{p}} \leq C_{1}$ for $p \in\left(p_{0}, 1\right]$.

Proof. First notice that, if $(2.9)$ a is true for $p_{0} \in(0,1]$, then it holds true with $p_{0}=1$ by the $p$-triangle inequality. Further

$$
\begin{aligned}
\|A a\|_{p_{0}, \mathrm{w}_{2}}^{p_{0}} & =\sum_{\lambda \in \Lambda}\left|\sum_{\lambda^{\prime} \in \Lambda} A_{\lambda, \lambda^{\prime}} a_{\lambda^{\prime}}\right|^{p_{0}} \mathrm{w}_{2}(\lambda)^{p_{0}} \\
& \leq \sum_{\lambda \in \Lambda} \sum_{\lambda^{\prime} \in \Lambda}\left|A_{\lambda, \lambda^{\prime}}\right|^{p_{0}}\left|a_{\lambda^{\prime}}\right|^{p_{0}} \mathrm{w}_{2}(\lambda)^{p_{0}} \\
& =\sum_{\lambda^{\prime} \in \Lambda}\left|a_{\lambda^{\prime}}\right|^{p_{0}} \sum_{\lambda \in \Lambda} \mathrm{w}_{2}(\lambda)^{p_{0}}\left|A_{\lambda, \lambda^{\prime}}\right|^{p_{0}} \\
& \leq C_{1}^{p_{0}} \sum_{\lambda^{\prime} \in \Lambda}\left|a_{\lambda^{\prime}}\right|^{p_{0}} \mathrm{w}_{1}\left(\lambda^{\prime}\right)^{p_{0}} \\
& =C_{1}^{p_{0}}\|a\|_{p_{0}, \mathrm{w}_{1}}^{p_{0}} .
\end{aligned}
$$


Therefore, the interpolation theorem ([25, Corollary 2.2]) yields item (国). Now, assuming [2.9)b we can estimate

$$
\begin{aligned}
\|A a\|_{\infty, w_{2}} & \leq \sup _{\lambda \in \Lambda} \sum_{\lambda^{\prime} \in \Lambda}\left|A_{\lambda, \lambda^{\prime}}\right|\left|a_{\lambda^{\prime}}\right| \mathrm{w}_{2}(\lambda) \\
& =\sup _{\lambda \in \Lambda} \mathrm{w}_{2}(\lambda) \sum_{\lambda^{\prime} \in \Lambda}\left|A_{\lambda, \lambda^{\prime}}\right| \mathrm{w}_{1}\left(\lambda^{\prime}\right)^{-1}\left|a_{\lambda^{\prime}}\right| \mathrm{w}_{1}\left(\lambda^{\prime}\right) \\
& \leq \sup _{\lambda \in \Lambda} \mathrm{w}_{2}(\lambda) \sum_{\lambda^{\prime} \in \Lambda}\left|A_{\lambda, \lambda^{\prime}}\right| \mathrm{w}_{1}\left(\lambda^{\prime}\right)^{-1} \sup _{\lambda^{\prime} \in \Lambda}\left|a_{\lambda^{\prime}}\right| \mathrm{w}_{1}\left(\lambda^{\prime}\right) \\
& \leq C_{2} \sup _{\lambda \in \Lambda} \mathrm{w}_{2}(\lambda) \mathrm{w}_{2}(\lambda)^{-1} \sup _{\lambda^{\prime} \in \Lambda}\left|a_{\lambda^{\prime}}\right| \mathrm{w}_{1}\left(\lambda^{\prime}\right) \\
& =C_{2}\|a\|_{\infty, w_{1}},
\end{aligned}
$$

whence we obtain item (b). Finally assume both (2.9)a and (2.9)b Then, by the interpolation theorem (25, Corollary 2.2]), items (国) and (b) imply item (ㅁ).

The estimate for $\|A\|_{\ell_{w_{1}}^{p} \rightarrow \ell_{w_{2}}^{p}}$ with $p \in\left(p_{0}, 1\right]$ follows from [25, Proposition 1.1, Theorem 2.4], interpolating between $p_{0}$ and 1 . As for the case $p \in[1, \infty]$, the bound follows easily by applying the Hölder inequality, (2.9)b and (2.9)a with $p_{0}=1$ (see [24, Lemma 4]).

If a matrix $A \in \mathbb{C}^{\Lambda \times \Lambda}$ decays with respect to some bounding function (e.g an index distance),

$$
\left|A_{\lambda, \lambda^{\prime}}\right| \lesssim \omega\left(\lambda, \lambda^{\prime}\right)^{-N}
$$

one can test the boundedness of $A$ by testing estimates of the form

$$
\sum_{\lambda \in \Lambda} \mathrm{w}_{2}^{p_{0}}(\lambda) \omega\left(\lambda, \lambda^{\prime}\right)^{-p_{0} K} \lesssim \mathrm{w}_{1}^{p_{0}}\left(\lambda^{\prime}\right), \quad \sum_{\lambda^{\prime} \in \Lambda} \omega\left(\lambda, \lambda^{\prime}\right)^{-K} \mathrm{w}_{1}^{-1}\left(\lambda^{\prime}\right) \lesssim \mathrm{w}_{2}^{-1}(\lambda)
$$

which imply conditions (2.9) for $N$ sufficiently large. In oder to give a precise statement, we introduce the concept of admissibility with respect to two weight sequences $\mathrm{w}_{1}, \mathrm{w}_{2}$ and a root $p_{0}$.

(2.10) Definition. Let $\mathrm{w}_{1}, \mathrm{w}_{2}: \Lambda \rightarrow(0,+\infty), p_{0} \in(0,1]$ and $K \geq 1$. An index distance $\omega$ is called $\left(\mathrm{w}_{1}, \mathrm{w}_{2}, p_{0}, K\right)$-admissible if

$$
\sum_{\lambda \in \Lambda} \mathrm{w}_{2}^{p_{0}}(\lambda) \omega\left(\lambda, \lambda^{\prime}\right)^{-p_{0} K} \leq C_{1}^{p_{0}} \mathbf{w}_{1}^{p_{0}}\left(\lambda^{\prime}\right), \quad \sum_{\lambda^{\prime} \in \Lambda} \omega\left(\lambda, \lambda^{\prime}\right)^{-K} \mathbf{w}_{1}^{-1}\left(\lambda^{\prime}\right) \leq C_{2} \mathbf{w}_{2}^{-1}(\lambda),
$$

for some $C_{1}, C_{2}>0$.

Note that, thanks to property (2.1) (iii), $K$-admissibility as defined in (2.3) is equivalent to $(1,1,1, K)$ admissibility as defined in (2.10)

(2.11) Proposition. Let $\omega$ be a $\left(\mathrm{w}_{1}, \mathrm{w}_{2}, p_{0}, K\right)$-admissible index distance. If $A \in \mathcal{B}_{N}$ for some $N \geq K$, then it defines a bounded operator from $\ell_{\mathrm{w}_{1}}^{p}(\Lambda)$ to $\ell_{\mathrm{w}_{2}}^{p}(\Lambda)$ for all $p \in\left[p_{0}, \infty\right]$, with

$$
\begin{aligned}
\|A\|_{\ell_{w_{1}}^{p} \rightarrow \ell_{\mathrm{w}_{2}}^{p}} & \leq C_{1}^{1 / p} C_{2}^{1 / p^{\prime}}\|A\|_{\mathcal{B}_{N}} \quad p \in[1, \infty], \\
\|A\|_{\ell_{w_{1}}^{p} \rightarrow \ell_{\mathrm{w}_{2}}^{p}} & \leq C_{1}\|A\|_{\mathcal{B}_{N}} \quad p \in\left(p_{0}, 1\right] .
\end{aligned}
$$

If $\omega$ is $K$-admissible, then $A$ defines a bounded operator from $\ell^{p}(\Lambda)$ to $\ell^{p}(\Lambda)$ for all $p \in[1, \infty]$, with

$$
\|A\|_{\ell^{p} \rightarrow \ell^{p}} \leq C_{S}^{N / p} C_{\omega}\|A\|_{\mathcal{B}_{N}} \quad p \in[1, \infty] .
$$

Proof. The proof follows directly by applying Lemma (2.8).

\subsection{Inverse Closedness}

For several applications it is important to know the localization properties of the operator $A^{-1}$, assuming that $A$, restricted to its image, constitutes an isomorphism $A: \ell^{2}(\Lambda) \rightarrow \ell^{2}(\Lambda)$. For instance, as we have seen in the previous subsection, if it can be shown that $A^{-1} \in \mathcal{B}_{N}$ for sufficiently large $N$ one can deduce the boundedness of $A^{-1}$ on a large class of sequence spaces. Except for very special cases of $\omega$ it cannot be expected that $A^{-1} \in \mathcal{B}_{N}$ if $A \in \mathcal{B}_{N}$. However, we shall show that the Moore-Penrose pseudoinverse $A^{+} \in \mathcal{B}_{N^{+}}$whenever $A \in \mathcal{B}_{N}$, where $N^{+} \leq N$, depending only on $\omega$ and the spectrum of $A$. Moreover, this dependence will be made completely explicit.

We now describe the spectral assumption on $A$, which we shall impose in our analysis. 
(2.12) Definition. The matrix $A$ viewed as an operator from $\ell^{2}(\Lambda)$ to itself possesses a spectral gap if there exist numbers $0<a \leq b<\infty$ such that

$$
\sigma_{2}(A) \subset\{0\} \cup[a, b],
$$

where $\sigma_{2}(A)$ denotes the $\ell^{2}(\Lambda)$-spectrum of $A$.

It $A$ is symmetric and possesses a spectral gap we can define its Moore-Penrose pseudoinverse $A^{+}$which satisfies the normal equations

$$
A^{2} A^{+}=A \text {. }
$$

Having stated all necessary definitions we can now state our main result.

(2.14) Theorem. Assume that $A \in \mathcal{B}_{N+L}$ with

$$
N \geq K \quad L \geq \max \left(2 N \log _{C_{\Lambda}} C_{T}, 2 K\right)
$$

is symmetric and possesses a spectral gap, e.g.,

$$
\sigma_{2}(A) \subset\{0\} \cup[a, b] .
$$

Then with $A^{+}$denoting its Moore-Penrose pseudoinverse we have

$$
A^{+} \in \mathcal{B}_{N^{+}}
$$

with

$$
N^{+}=N\left(1-\frac{\log \left(1+\frac{2}{a^{2}+b^{2}}\|A\|_{\mathcal{B}_{N+L}}^{2}\left(1+C_{\omega}\right)^{2}\right)}{\log \left(\frac{b^{2}-a^{2}}{b^{2}+a^{2}}\right)}\right)^{-1} .
$$

Proof. The proof goes exactly as the proof of [21, Theorem 2.12], using our submultiplicativity result, Theorem (2.7),

\section{Application to $\alpha$-Molecules}

We intend to apply the general results of the previous section to the study of $\alpha$-molecules 22. This class of systems includes as special cases wavelets, curvelets, shearlets, hybrid shearlets and ridgelets, therefore our results will allow us to gain localization results for all these systems simultaneously. We proceed as follows. In Subsection 3.1 we describe the index distance $\omega$ which has been introduced in 22] and which is defined on a contiuous phase space $P$. Then we prove that this function $\omega$ satisfies all the assumptions of Definition (2.1) This turns out to be the most technical part of this work. Then, only later in Subsection 3.2 we briefly introduce the notion of $\alpha$-molecules. In a system of $\alpha$-molecules, every function is associated with a point in the phase space $P$ and therefore every such system is associated to a discrete sampling set $\Lambda \subset P$. We discuss two canonical choices of $\Lambda$ in detail: so-called curvelet-type systems in Subsubsection 3.2.1 and so-called shearlet-type systems in Subsubsection 3.2.2 In both cases we show that the index distance $\omega$ restricted to $\Lambda$ is separated and admissible. In summary $\alpha$-molecules, together with the index distance introduced in 22, fits into the abstract framework developed earlier in Section 2, As an application we present localization for canonical duals of frames of $\alpha$-curvelets (Theorem [3.17) and $\alpha$-shearlets (Theorem (3.23)].

\subsection{Index Distance}

Before we describe the notion of $\alpha$-molecules we start by defining the corresponding index distance $\omega_{\alpha}$ and show that our main result can indeed be applied to this index distance. Roughly speaking, $\alpha$-molecules can be associated with a scale, an orientation and a location. Therefore we first define a contiuous parameter space $P$ as the product

$$
P:=\mathbb{R}_{+} \times S^{1} \times \mathbb{R}^{2}
$$


The parameters in $P$ will be denoted by $p=(s, \theta, x), p^{\prime}=\left(s^{\prime}, \theta^{\prime}, x^{\prime}\right)$, and so on. We also define, for each $\alpha \in[0,1]$, the function

$$
\omega_{\alpha}:=M\left(1+d_{\alpha}\right)
$$

where

$$
\begin{aligned}
& M\left(p, p^{\prime}\right):=\max \left(s / s^{\prime}, s^{\prime} / s\right) \\
& d_{\alpha}\left(p, p^{\prime}\right):=\min \left(s, s^{\prime}\right)^{2(1-\alpha)}\left|\theta-\theta^{\prime}\right|^{2}+\min \left(s, s^{\prime}\right)^{2 \alpha}\left\|x-x^{\prime}\right\|^{2}+\min \left(s, s^{\prime}\right)\left|\left\langle x-x^{\prime}, e_{\theta}\right\rangle\right|,
\end{aligned}
$$

$e_{\theta}$ being the "co-direction" $(\cos \theta,-\sin \theta)$. We shall often adopt the abbreviations $\Delta \theta:=\theta-\theta^{\prime}$ and $\Delta x:=x-x^{\prime}$.

(3.3) Remark. This definition of $\omega_{\alpha}$ differs from the one presented in 22 , where the last term of $d_{\alpha}\left(p, p^{\prime}\right)$ is replaced by

$$
\frac{\min \left(s, s^{\prime}\right)^{2}\left|\left\langle\Delta x, e_{\theta}\right\rangle\right|^{2}}{1+\min \left(s, s^{\prime}\right)^{2(1-\alpha)}|\Delta \theta|^{2}}
$$

However, an application of the inequality of arithmetic and geometric means yields

$$
\begin{aligned}
& 1+\min \left(s, s^{\prime}\right)^{2(1-\alpha)}|\Delta \theta|^{2}+\frac{\min \left(s, s^{\prime}\right)^{2}\left|\left\langle\Delta x, e_{\theta}\right\rangle\right|^{2}}{1+\min \left(s, s^{\prime}\right)^{2(1-\alpha)}|\Delta \theta|^{2}} \\
= & \left(\sqrt{1+\min \left(s, s^{\prime}\right)^{2(1-\alpha)}|\Delta \theta|^{2}}\right)^{2}+\left(\frac{\min \left(s, s^{\prime}\right)\left|\left\langle\Delta x, e_{\theta}\right\rangle\right|}{\sqrt{1+\min \left(s, s^{\prime}\right)^{2(1-\alpha)}|\Delta \theta|^{2}}}\right)^{2} \\
\geq & 2 \sqrt{1+\min \left(s, s^{\prime}\right)^{2(1-\alpha)}|\Delta \theta|^{2}} \frac{\min \left(s, s^{\prime}\right)\left|\left\langle\Delta x, e_{\theta}\right\rangle\right|}{\sqrt{1+\min \left(s, s^{\prime}\right)^{2(1-\alpha)}|\Delta \theta|^{2}}} \\
= & 2 \min \left(s, s^{\prime}\right)\left|\left\langle\Delta x, e_{\theta}\right\rangle\right| ;
\end{aligned}
$$

then, if we call $\tilde{\omega}_{\alpha}$ the index distance introduced in [22, we have $\tilde{\omega}_{\alpha} \geq 2 \omega_{\alpha}$. Now, the key concept to preserve here is almost orthogonality (see [22] for details), and this inequality shows exactly that systems which are almost orthogonal respect to $\tilde{\omega}_{\alpha}$ are still almost orthogonal respect to $\omega_{\alpha}$.

Also note that, for suitable choices of the parameters, $\omega_{1}$ corresponds to the wavelet index distance, whereas $\omega_{\frac{1}{2}}$ returns the curvelet-shearlet index distance studied in [21].

The restriction of $\omega_{\alpha}$ to any discrete index set $\Lambda \subset P$ describes an index distance as we show in the next result.

(3.4) Proposition. $\omega_{\alpha}$ is an index distance for all $\alpha \in[0,1]$ and all discrete index sets $\Lambda \subset P$. The resulting constants obey $C_{S} \leq 2$ and $C_{T} \leq 4$.

Proof. For ease of notation, we shall avoid to specify the index $\alpha$ in $\omega_{\alpha}$. It is apparent that $\omega$ is 1 on the diagonal, that is property (2.1) (i). We next prove properties (2.1) (iii) and (iii).

(2.1) (iii). Notice that the only non symmetric term in $\omega\left(p, p^{\prime}\right)$ is $\min \left(s, s^{\prime}\right)\left|\left\langle\Delta x, e_{\theta}\right\rangle\right|$, so it sufficies to show that $\left|\left\langle\Delta x, e_{\theta}\right\rangle\right| \lesssim \min \left(s, s^{\prime}\right)^{-1} d\left(p^{\prime}, p\right)$. Since

$$
\left|\left\langle\Delta x, e_{\theta}\right\rangle\right| \leq\left|\left\langle\Delta x, e_{\theta}\right\rangle\right|+\left|\left\langle\Delta x, e_{\theta^{\prime}}\right\rangle\right| \leq\left|\left\langle\Delta x, e_{\theta}\right\rangle-\left\langle\Delta x, e_{\theta^{\prime}}\right\rangle\right|+2\left|\left\langle\Delta x, e_{\theta^{\prime}}\right\rangle\right|,
$$

it remains to estimate

$$
\left|\left\langle\Delta x, e_{\theta}\right\rangle-\left\langle\Delta x, e_{\theta^{\prime}}\right\rangle\right|=\left|\left\langle\Delta x, e_{\theta}-e_{\theta^{\prime}}\right\rangle\right| \leq\left\|e_{\theta}-e_{\theta^{\prime}}\right\|\|\Delta x\| .
$$

By prosthaphaeresis

$$
e_{\theta}-e_{\theta^{\prime}}=\left(\cos \theta-\cos \theta^{\prime}, \sin \theta-\sin \theta^{\prime}\right)=2 \sin \left(\frac{\theta-\theta^{\prime}}{2}\right)\left(-\sin \left(\frac{\theta+\theta^{\prime}}{2}\right), \cos \left(\frac{\theta+\theta^{\prime}}{2}\right)\right),
$$

then

$$
\left\|e_{\theta}-e_{\theta^{\prime}}\right\|=2\left|\sin \left(\frac{\theta-\theta^{\prime}}{2}\right)\right| \leq 2\left|\frac{\theta-\theta^{\prime}}{2}\right|=\left|\theta-\theta^{\prime}\right|
$$


Therefore

$$
\begin{aligned}
\left|\left\langle\Delta x, e_{\theta}\right\rangle-\left\langle\Delta x, e_{\theta^{\prime}}\right\rangle\right| & \leq|\Delta \theta|\|\Delta x\| \\
& =\min \left(s, s^{\prime}\right)^{1 / 2-\alpha}|\Delta \theta| \min \left(s, s^{\prime}\right)^{\alpha-1 / 2}\|\Delta x\| \\
& \leq \frac{1}{2}\left(\min \left(s, s^{\prime}\right)^{1-2 \alpha}|\Delta \theta|^{2}+\min \left(s, s^{\prime}\right)^{2 \alpha-1}\|\Delta x\|^{2}\right) \\
& \leq \frac{1}{2} \min \left(s, s^{\prime}\right)^{-1} d\left(p, p^{\prime}\right)
\end{aligned}
$$

by the inequality of arithmetic and geometric means. Thus we get (2.1) (ii) with bound $C_{S} \leq 2$.

(2.1) (iii). We shall write indices 01 for the expressions evaluated in $\left(p, p^{\prime}\right), 02$ for $\left(p, p^{\prime \prime}\right)$ and 21 for $\left(p^{\prime \prime}, p^{\prime}\right)$. The letter $s_{01}$ will be a short for $\min \left(s, s^{\prime}\right)$, and similarly with regard to $s_{02}$ and $s_{21}$.

Well then, we have to show that $\omega_{01} \leq C \omega_{02} \omega_{21}$ for some constant $C \geq 1$. Write

$$
\begin{aligned}
\omega_{01} & =M_{01}\left(1+d_{01}\right) \\
& =M_{01}\left(1+s_{01}^{2-2 \alpha} \Delta \theta^{2}+s_{01}^{2 \alpha} \Delta x^{2}+s_{01}\left|\left\langle\Delta x, e_{\theta}\right\rangle\right|\right) \\
& =\underbrace{M_{01}\left(s_{01}^{2-2 \alpha} \Delta \theta^{2}+s_{01}^{2 \alpha} \Delta x^{2}\right)}_{=: A}+\underbrace{M_{01}\left(1+s_{01}\left|\left\langle\Delta x, e_{\theta}\right\rangle\right|\right)}_{=: B} .
\end{aligned}
$$

We shall prove that

$$
\begin{aligned}
& A \leq 2 M_{02} M_{21}\left(d_{02}+d_{21}\right) \leq 2 \omega_{02} \omega_{21}, \\
& B \leq 2 M_{02} M_{21}\left(1+d_{02}+d_{21}+d_{02} d_{21}\right)=2 \omega_{02} \omega_{21},
\end{aligned}
$$

so that $\omega_{01}=A+B \leq 4 \omega_{02} \omega_{21}$, as we claim.

In order to verify (3.5) and (3.6), we first observe that $\omega$ is translational invariant, namely

$$
\omega\left((s, \theta, x),\left(s^{\prime}, \theta^{\prime}, x^{\prime}\right)\right)=\omega\left((s, \theta, x+t),\left(s^{\prime}, \theta^{\prime}, x^{\prime}+t\right)\right) \quad \forall t \in \mathbb{R}^{2},
$$

and therefore we can set $x=0$. Moreover, we can work in coordinates $e_{\theta}, e_{\theta}^{\perp}$, so that $e_{\theta}=(1,0)$ and $\theta=0$. Coordinates for $x^{\prime}$ and $x^{\prime \prime}$ are called $\left(x_{1}, y_{1}\right)$ and $\left(x_{2}, y_{2}\right)$, respectively. With this choices we have:

$$
\begin{aligned}
& d_{01}=s_{01}^{2-2 \alpha}\left|\theta^{\prime}\right|^{2}+s_{01}^{2 \alpha}\left(\left|x_{1}\right|^{2}+\left|y_{1}\right|^{2}\right)+s_{01}\left|x_{1}\right|, \\
& d_{02}=s_{02}^{2-2 \alpha}\left|\theta^{\prime \prime}\right|^{2}+s_{02}^{2 \alpha}\left(\left|x_{2}\right|^{2}+\left|y_{2}\right|^{2}\right)+s_{02}\left|x_{2}\right|, \\
& d_{21}=s_{21}^{2-2 \alpha}\left|\theta^{\prime \prime}-\theta^{\prime}\right|^{2}+s_{21}^{2 \alpha}\left(\left|x_{2}-x_{1}\right|^{2}+\left|y_{2}-y_{1}\right|^{2}\right)+s_{21}\left|\cos \theta^{\prime \prime}\left(x_{2}-x_{1}\right)+\sin \theta^{\prime \prime}\left(y_{2}-y_{1}\right)\right| .
\end{aligned}
$$

Let us start with (3.5). We estimate

$$
\begin{aligned}
M_{01} s_{01}^{2-2 \alpha}\left|\theta^{\prime}\right|^{2} & \leq 2\left(M_{01} s_{01}^{2-2 \alpha}\left|\theta^{\prime \prime}\right|^{2}+M_{01} s_{01}^{2-2 \alpha}\left|\theta^{\prime \prime}-\theta^{\prime}\right|^{2}\right) \\
& \leq 2\left(M_{02} M_{21} s_{02}^{2-2 \alpha}\left|\theta^{\prime \prime}\right|^{2}+M_{02} M_{21} s_{21}^{2-2 \alpha}\left|\theta^{\prime \prime}-\theta^{\prime}\right|^{2}\right)
\end{aligned}
$$

by Lemma (A.1) (just exponentiate the appropriate inequality to use it in multiplicative form). Likewise we have

$$
\begin{aligned}
M_{01} s_{01}^{2 \alpha}\left\|x^{\prime}\right\|^{2} & \leq 2\left(M_{01} s_{01}^{2 \alpha}\left\|x^{\prime \prime}\right\|^{2}+M_{01} s_{01}^{2 \alpha}\left\|x^{\prime \prime}-x^{\prime}\right\|^{2}\right) \\
& \leq 2\left(M_{02} M_{21} s_{02}^{2 \alpha}\left\|x^{\prime \prime}\right\|^{2}+M_{02} M_{21} s_{21}^{2 \alpha}\left\|x^{\prime \prime}-x^{\prime}\right\|^{2}\right),
\end{aligned}
$$

again by (A.1) (in multiplicative form). Thus we get (3.5)

Now we move on to (3.6). One has

$$
B=M_{01}+M_{01} s_{01}\left|x_{1}\right| \leq M_{02} M_{21}+M_{01} s_{01}\left|x_{1}\right|
$$

then, if we show that

$$
\underbrace{M_{01} s_{01}\left|x_{1}\right|}_{=: L} \leq \underbrace{M_{02} M_{21}\left[1+2\left(d_{02}+d_{21}+d_{02} d_{21}\right)\right]}_{=: R},
$$

we have (3.6) First notice that

$$
\begin{aligned}
L & =\frac{\max \left(s, s^{\prime}\right)}{\min \left(s, s^{\prime}\right)} \min \left(s, s^{\prime}\right)\left|x_{1}\right| \\
& =\max \left(s, s^{\prime}\right)\left|x_{1}\right| .
\end{aligned}
$$

Our estimates for $R$ depend on how large is the angle $\theta^{\prime \prime}$. We shall occasionally write $s_{012}$ for $\min \left(s, s^{\prime}, s^{\prime \prime}\right)$. 
$\left|\theta^{\prime \prime}\right| \geq \pi / 4$. We have

$$
\begin{aligned}
R & \geq M_{02} M_{21}\left[1+2\left(s_{02}\left|x_{2}\right|+s_{02}^{2-2 \alpha}\left|\theta^{\prime \prime}\right|^{2} s_{21}^{2 \alpha}\left|x_{2}-x_{1}\right|^{2}\right)\right] \\
& \geq M_{02} M_{21}\left[1+2\left(s_{02}\left|x_{2}\right|+\frac{\pi^{2}}{16} s_{02}^{2-2 \alpha} s_{21}^{2 \alpha}\left|x_{2}-x_{1}\right|^{2}\right)\right] \\
& \geq M_{02} M_{21}\left[1+2\left(s_{012}\left|x_{2}\right|+\frac{\pi^{2}}{16} s_{012}^{2}\left|x_{2}-x_{1}\right|^{2}\right)\right]=: R^{\prime}
\end{aligned}
$$

Dividing by $M_{02} M_{21}$, we have that $R \geq L$ if

$$
1+2 s_{012}\left|x_{2}\right|+\frac{\pi^{2}}{8} s_{012}^{2}\left|x_{2}-x_{1}\right|^{2} \geq \frac{\max \left(s, s^{\prime}\right)}{M_{02} M_{21}}\left|x_{1}\right| .
$$

But

$$
\begin{aligned}
\frac{\max \left(s, s^{\prime}\right)}{M_{02} M_{21}} & =\max \left(s, s^{\prime}\right) \frac{\min \left(s, s^{\prime \prime}\right)}{\max \left(s, s^{\prime \prime}\right)} \frac{\min \left(s^{\prime \prime}, s^{\prime}\right)}{\max \left(s^{\prime \prime}, s^{\prime}\right)} \\
& =\max \left(s, s^{\prime}\right) \frac{\max \left(\min \left(s, s^{\prime \prime}\right), \min \left(s^{\prime \prime}, s^{\prime}\right)\right) \min \left(s, s^{\prime}, s^{\prime \prime}\right)}{\min \left(\max \left(s, s^{\prime \prime}\right), \max \left(s^{\prime \prime}, s^{\prime}\right)\right) \max \left(s, s^{\prime}, s^{\prime \prime}\right)} \\
& \leq \max \left(s, s^{\prime}\right) \frac{\min \left(s, s^{\prime}, s^{\prime \prime}\right)}{\max \left(s, s^{\prime}, s^{\prime \prime}\right)} \quad \text { by Lemma (A.2) } \\
& \leq \max \left(s, s^{\prime}, s^{\prime \prime}\right) \frac{\min \left(s, s^{\prime}, s^{\prime \prime}\right)}{\max \left(s, s^{\prime}, s^{\prime \prime}\right)} \\
& =\min \left(s, s^{\prime}, s^{\prime \prime}\right)=s_{012},
\end{aligned}
$$

whence $R \geq L$ provided that

$$
1+2 a\left|x_{2}\right|+\frac{\pi^{2}}{8} a^{2}\left|x_{2}-x_{1}\right|^{2} \geq a\left|x_{1}\right|
$$

for every $a>0$ and $x_{1}, x_{2} \in \mathbb{R}$. It is actually equivalent to show this for $a=1$, since we can always replace $\left(x_{1}, x_{2}\right)$ with $\left(a x_{1}, a x_{2}\right)$. By the usual triangle inequality, we have

$$
\left|x_{1}\right| \leq\left|x_{2}-x_{1}\right|+\left|x_{2}\right|
$$

Now, if $\left|x_{2}-x_{1}\right| \geq 1$, then $\left|x_{2}-x_{1}\right| \leq\left|x_{2}-x_{1}\right|^{2}$ and we are done. Otherwise $\left|x_{2}-x_{1}\right| \leq 1$, and we are done as well.

$\left|\theta^{\prime \prime}\right| \leq \pi / 4$. We have

$$
\begin{aligned}
R & \geq M_{02} M_{21}\left\{1+2\left[s_{02}\left|x_{2}\right|+s_{21}\left|\cos \theta^{\prime \prime}\left(x_{2}-x_{1}\right)-\sin \theta^{\prime \prime}\left(y_{2}-y_{1}\right)\right|+s_{02}^{2-2 \alpha}\left|\theta^{\prime \prime}\right|^{2} s_{21}^{2 \alpha}\left|y_{2}-y_{1}\right|^{2}\right]\right\} \\
& \geq M_{02} M_{21}\left\{1+2\left[s_{012}\left|x_{2}\right|+s_{012}\left|\cos \theta^{\prime \prime}\left(x_{2}-x_{1}\right)-\sin \theta^{\prime \prime}\left(y_{2}-y_{1}\right)\right|+s_{012}^{2}\left|\theta^{\prime \prime}\right|^{2}\left|y_{2}-y_{1}\right|^{2}\right]\right\} \\
& \left.\geq M_{02} M_{21}\left\{1+2\left[s_{012}\left|x_{2}\right|+s_{012}\left(\cos \theta^{\prime \prime}\left|x_{2}-x_{1}\right|-\sin \left|\theta^{\prime \prime}\right|\left|y_{2}-y_{1}\right|\right)+s_{012}^{2}\left|\theta^{\prime \prime}\right|^{2}\left|y_{2}-y_{1}\right|^{2}\right)\right]\right\} \\
& \geq M_{02} M_{21}\left\{1+2 s_{012}\left|x_{2}\right|+\sqrt{2} s_{012}\left|x_{2}-x_{1}\right|-2 s_{012} \sin \left|\theta^{\prime \prime}\right|\left|y_{2}-y_{1}\right|+2 s_{012}^{2}\left|\theta^{\prime \prime}\right|^{2}\left|y_{2}-y_{1}\right|^{2}\right\} \\
& =M_{02} M_{21} \underbrace{\left(1-2 s_{012} \sin \left|\theta^{\prime \prime}\right|\left|y_{2}-y_{1}\right|+2 s_{012}^{2}\left|\theta^{\prime \prime}\right|^{2}\left|y_{2}-y_{1}\right|^{2}\right)}_{=: R_{1}} \\
& +M_{02} M_{21}\left(2 s_{012}\left|x_{2}\right|+\sqrt{2} s_{012}\left|x_{2}-x_{1}\right|\right) \\
& \geq M_{02} M_{21} R_{1}+\underbrace{M_{02} M_{21} s_{012}\left(\left|x_{2}\right|+\left|x_{2}-x_{1}\right|\right)}_{=: R_{2}} .
\end{aligned}
$$

In $R_{1}$ we can regard $s_{012}$ as any $a>0$, and we can actually set $a=1$ by replacing $\left(y_{1}, y_{2}\right)$ with $\left(a y_{1}, a y_{2}\right)$. Thus we get a polynomial in $\left|y_{2}-y_{1}\right|$ with discriminant

$$
\Delta / 4=\sin ^{2}\left|\theta^{\prime \prime}\right|-2\left|\theta^{\prime \prime}\right|^{2} \leq 0,
$$

whence $R_{1} \geq 0$. It follows that $R \geq R_{2}$. 
On the other hand, we have

$$
\begin{aligned}
M_{02} M_{21} s_{012} & =\frac{\max \left(s, s^{\prime \prime}\right)}{\min \left(s, s^{\prime \prime}\right)} \frac{\max \left(s^{\prime \prime}, s^{\prime}\right)}{\min \left(s^{\prime \prime}, s^{\prime}\right)} \min \left(s, s^{\prime}, s^{\prime \prime}\right) \\
& =\frac{\max \left(s, s^{\prime}, s^{\prime \prime}\right) \min \left(\max \left(s, s^{\prime \prime}\right), \max \left(s^{\prime \prime}, s^{\prime}\right)\right)}{\min \left(s, s^{\prime}, s^{\prime \prime}\right) \max \left(\min \left(s, s^{\prime \prime}\right), \min \left(s^{\prime \prime}, s^{\prime}\right)\right)} \min \left(s, s^{\prime}, s^{\prime \prime}\right) \\
& =\max \left(s, s^{\prime}, s^{\prime \prime}\right) \frac{\min \left(\max \left(s, s^{\prime \prime}\right), \max \left(s^{\prime \prime}, s^{\prime}\right)\right)}{\max \left(\min \left(s, s^{\prime \prime}\right), \min \left(s^{\prime \prime}, s^{\prime}\right)\right)} \\
& \geq \max \left(s, s^{\prime}, s^{\prime \prime}\right) \quad \text { by Lemma (A.2) } \\
& \geq \max \left(s, s^{\prime}\right),
\end{aligned}
$$

whence

$$
\begin{aligned}
R_{2} & \geq \max \left(s, s^{\prime}\right)\left(\left|x_{2}\right|+\left|x_{2}-x_{1}\right|\right) \\
& \geq \max \left(s, s^{\prime}\right)\left|x_{1}\right|=L
\end{aligned}
$$

The property (2.1) iiii) is finally proven, with constant bound $C_{T} \leq 4$.

(3.7) Remark. Following [21], one may think to write $\omega_{\alpha}\left(p, p^{\prime}\right)=\max \left(s, s^{\prime}\right)\left(1+\min \left(s, s^{\prime}\right) \tilde{d}_{\alpha}\left(p, p^{\prime}\right)\right)$, with

$$
\tilde{d}_{\alpha}\left(p, p^{\prime}\right):=\min \left(s, s^{\prime}\right)^{1-2 \alpha}|\Delta \theta|^{2}+\min \left(s, s^{\prime}\right)^{2 \alpha-1}\|\Delta x\|^{2}+\left|\left\langle\Delta x, e_{\theta}\right\rangle\right|,
$$

and check the assumptions made in 21. It turns out that all the hypothesis are satisfied, except for the very important pseudo-triangle inequality

$$
\tilde{d}_{\alpha}\left(p, p^{\prime}\right) \lesssim \tilde{d}_{\alpha}\left(p, p^{\prime \prime}\right)+\tilde{d}_{\alpha}\left(p^{\prime \prime}, p^{\prime}\right)
$$

which is true if and only if $\alpha=\frac{1}{2}$. To see this, begin by fixing $\alpha \in\left[0, \frac{1}{2}\right)$, so that $1-2 \alpha \in(0,1]$. For any $C \geq 1$ pick

$$
s=s^{\prime}>C^{1 /(1-2 \alpha)}, \quad s^{\prime \prime}=1, \quad \theta=0, \quad \theta^{\prime}=\theta^{\prime \prime} \neq 0, \quad x=x^{\prime}=x^{\prime \prime}=0,
$$

whence we obtain

$$
\tilde{d}_{\alpha}\left(p, p^{\prime}\right)=s^{1-2 \alpha} \theta^{\prime 2}>C \theta^{\prime 2}=C \tilde{d}_{\alpha}\left(p, p^{\prime \prime}\right)=C\left(\tilde{d}_{\alpha}\left(p, p^{\prime \prime}\right)+\tilde{d}_{\alpha}\left(p^{\prime \prime}, p^{\prime}\right)\right) .
$$

Similarly, if $\alpha \in\left(\frac{1}{2}, 1\right], 2 \alpha-1 \in(0,1]$, for any $C \geq 1$ we can set

$$
s=s^{\prime}>C^{1 /(2 \alpha-1)}, \quad s^{\prime \prime}=1, \quad \theta=\theta^{\prime}=\theta^{\prime \prime}=0, \quad x=0, \quad x^{\prime}=x^{\prime \prime}=(0, y) \neq 0,
$$

whence

$$
\tilde{d}_{\alpha}\left(p, p^{\prime}\right)=s^{2 \alpha-1} y^{2}>C y^{2}=C \tilde{d}_{\alpha}\left(p, p^{\prime \prime}\right)=C\left(\tilde{d}_{\alpha}\left(p, p^{\prime \prime}\right)+\tilde{d}_{\alpha}\left(p^{\prime \prime}, p^{\prime}\right)\right) .
$$

\section{$3.2 \alpha$-Molecules}

We now introduce the notion of $\alpha$-molecules as in 22. There, $\alpha$-molecules are defined as systems of functions $\left(m_{\lambda}\right)_{\lambda \in \Lambda}$, where each $m_{\lambda} \in L^{2}\left(\mathbb{R}^{2}\right)$ has to satisfy some additional properties. In particular, each function $m_{\lambda}$ will be associated with a unique point in $P$, which is done via a parametrization as defined below.

(3.8) Definition. A parametrization consists of a pair $\left(\Lambda, \Phi_{\Lambda}\right)$ where $\Lambda$ is an index set and $\Phi_{\Lambda}$ is a mapping

$$
\Phi_{\Lambda}:\left\{\begin{array}{ccc}
\Lambda & \rightarrow & P \\
\lambda & \mapsto & \left(s_{\lambda}, \theta_{\lambda}, x_{\lambda}\right)
\end{array}\right.
$$

which associates with each $\lambda \in \Lambda$ a scale $s_{\lambda}$, a direction $\theta_{\lambda}$ and a location $x_{\lambda}$. $\omega \circ \Phi_{\Lambda}$.

With slight abuse of notation, below we shall confuse $\Lambda$ with the image $\Phi_{\Lambda}(\Lambda)$, and $\omega$ with the pull-back

Let

$$
D_{s}:=\left(\begin{array}{cc}
s & 0 \\
0 & s^{\alpha}
\end{array}\right), \quad R_{\theta}:=\left(\begin{array}{cc}
\cos \theta & -\sin \theta \\
\sin \theta & \cos \theta
\end{array}\right)
$$

denote respectively the anisotropic dilation matrix associated with $s>0$ and $\alpha \in[0,1]$ and the rotation matrix by an angle $\theta \in S^{1}$. Now we have collected all the necessary ingredients for defining $\alpha$-molecules. 
(3.10) Definition. Let $\left(\Lambda, \Phi_{\Lambda}\right)$ be a parametrization and $R, M, N_{1}, N_{2}>0$. A family $\left(m_{\lambda}\right)_{\lambda \in \Lambda} \subset L^{2}\left(\mathbb{R}^{2}\right)$ is called a family of $\alpha$-molecules with respect to $\left(\Lambda, \Phi_{\Lambda}\right)$ of order $\left(R, M, N_{1}, N_{2}\right)$, if it can be written as

$$
m_{\lambda}(x)=s_{\lambda}^{(1+\alpha) / 2} a^{(\lambda)}\left(D_{s_{\lambda}} R_{\theta_{\lambda}}\left(x-x_{\lambda}\right)\right)
$$

such that

$$
\left|\partial^{\beta} \hat{a}^{(\lambda)}(\xi)\right| \lesssim \min \left(1, s_{\lambda}^{-1}+\left|\xi_{1}\right|+s_{\lambda}^{-(1-\alpha)}\left|\xi_{2}\right|\right)^{M}\langle|\xi|\rangle^{-N_{1}}\left\langle\xi_{2}\right\rangle^{-N_{2}} \quad \text { for all }|\beta| \leq R .
$$

The implicit constants are uniform over $\lambda \in \Lambda$.

It is instructive to look at some special cases. For instance the case $\alpha=1$ corresponds to wavelet-type systems, whereas the case $\alpha=\frac{1}{2}$ corresponds to parabolic molecules [23, which include curvelets [14] and shearlets [29]. The case $\alpha=0$ corresponds to ridgelet-type systems [20, 9]. The systems with $\alpha \in\left(0, \frac{1}{2}\right)$ have been called 'hybrid' systems. Such systems, together with their approximation properties, have been studied recently in 27 .

Systems of $\alpha$-molecules are useful for the decomposition and reconstruction of functions $f \in L^{2}\left(\mathbb{R}^{2}\right)$ in a numerically stable fashion. To this end it is required that a system $\left(m_{\lambda}\right)_{\lambda \in \Lambda}$ constitues a frame in the sense that there exist constants $0<a \leq b<\infty$ such that

$$
a^{2}\|f\|_{L^{2}\left(\mathbb{R}^{2}\right)}^{2} \leq \sum_{\lambda \in \Lambda}\left|\left\langle f, m_{\lambda}\right\rangle_{L^{2}\left(\mathbb{R}^{2}\right)}\right|^{2} \leq b^{2}\|f\|_{L^{2}\left(\mathbb{R}^{2}\right)}^{2} \quad \text { for all } f \in L^{2}\left(\mathbb{R}^{2}\right) .
$$

If (3.11) holds true, there exists a canonical dual frame $\left(\tilde{m}_{\lambda}\right)_{\lambda \in \Lambda}$ satisfying

$$
f=\sum_{\lambda \in \Lambda}\left\langle f, m_{\lambda}\right\rangle_{L^{2}\left(\mathbb{R}^{2}\right)} \tilde{m}_{\lambda}=\sum_{\lambda \in \Lambda}\left\langle f, \tilde{m}_{\lambda}\right\rangle_{L^{2}\left(\mathbb{R}^{2}\right)} m_{\lambda} \quad \text { for all } f \in L^{2}\left(\mathbb{R}^{2}\right)
$$

see e.g. [11. Unless $a=b$, in which case $\tilde{m}_{\lambda}=a^{-2} m_{\lambda}$, the canonical dual frame is not in general explicitly known. Nevertheless, for a number of applications it is important to study its structure, in particular its similarity or dissimilarity to the primal frame $\left(m_{\lambda}\right)_{\lambda \in \Lambda}$. Again we refer to 21 for more detailed information. Crucial in this respect is the localization property which we define next.

(3.12) Definition. We say that a system $\left(m_{\lambda}\right)_{\lambda \in \Lambda}$ is $N$-localized (with respect to the index distance $(3.2)$ if such is its Gramian, that is

$$
\left(\left\langle m_{\lambda}, m_{\lambda^{\prime}}\right\rangle_{L^{2}\left(\mathbb{R}^{2}\right)}\right)_{\lambda, \lambda^{\prime} \in \Lambda} \in \mathcal{B}_{N}
$$

Notice that $\omega_{\alpha}$ provides a measure of the off-diagonal decay of the Gramian. In the following we shall study conditions under which the dual of a frame of $\alpha$-molecules is localized, provided that such is the primal frame.

In order to apply the machinery of Section 2 we first need to observe a couple of facts.

(3.13) Lemma. Given a frame $\left(m_{\lambda}\right)_{\lambda \in \Lambda} \subset L^{2}\left(\mathbb{R}^{2}\right)$ with frame constants a, b, the associated Gramian possesses the spectral gap

$$
\sigma_{2}\left(\left(\left\langle m_{\lambda}, m_{\lambda^{\prime}}\right\rangle_{L^{2}\left(\mathbb{R}^{2}\right)}\right)_{\lambda, \lambda^{\prime} \in \Lambda}\right) \subset\{0\} \cup[a, b] .
$$

Furthermore, the Moore-Penrose pseudoinverse of the Gramian $\left(\left\langle m_{\lambda}, m_{\lambda^{\prime}}\right\rangle_{L^{2}\left(\mathbb{R}^{2}\right)}\right)_{\lambda, \lambda^{\prime} \in \Lambda}$ is given by the dual Gramian $\left(\left\langle\tilde{m}_{\lambda}, \tilde{m}_{\lambda^{\prime}}\right\rangle_{L^{2}\left(\mathbb{R}^{2}\right)}\right)_{\lambda, \lambda^{\prime} \in \Lambda}$.

Proof. [21, Lemma 3.3].

In view of Lemma (3.13) if we can show that the index distance $\omega_{\alpha}$ restricted to a suitable discrete index set satisfies the assumptions of Section 2 we can directly appeal to Theorem $(2.14)$ to deduce localization results for the dual frame. The verification of these latter properties is the subject of the remainder of this section. In particular we shall consider curvelet-type and shearlet-type sampling sets below. 


\subsubsection{Curvelet-type Parametrization}

We start by considering curvelet-type parametrizations which arise by discretizing the scale parameter on a logarithmic scale and the directional parameter uniformly in polar angle (see 22 for more details). We show the admissibility and the separatedness of the resulting parametrization, which allows us to directly appeal to Theorem (2.14),

(3.14) Definition. Let $\alpha \in[0,1]$ and $g>1, \tau>0$ be some fixed parameters. Further, let $\left(\gamma_{j}\right)_{j \in \mathbb{N}}$ and $\left(L_{j}\right)_{j \in \mathbb{N}}$ be sequences of positive real numbers with $\gamma_{j} \asymp g^{-j(1-\alpha)}$, i.e. there are constants $C, c>0$ independent of $j$ such that $c g^{-j(1-\alpha)} \leq \gamma_{j} \leq C g^{-j(1-\alpha)}$, and $L_{j} \lesssim g^{j(1-\alpha)}$. An $\alpha$-curvelet parametrization is given by an index set of the form

$$
\Lambda_{\alpha}^{c}:=\left\{(j, l, k) \in \mathbb{N} \times \mathbb{Z} \times \mathbb{Z}^{2}:|l| \leq L_{j}\right\}
$$

and a mapping

$$
\Phi^{c}(\lambda):=\left(s_{\lambda}, \theta_{\lambda}, x_{\lambda}\right):=\left(g^{j}, l \gamma_{j}, R_{\theta_{\lambda}}^{-1} D_{s_{\lambda}}^{-1} \tau k\right) .
$$

The parameters $g>1$ and $\tau>0$ are sampling constants which determine the fineness of the sampling grid, $g$ for the scales and $\tau$ for locations.

Our next goal is to prove that the index distance $\omega_{\alpha}$ which arises from a curvelet-type parameterization separates the index set $\Lambda_{\alpha}^{c}$ and is admissible as defined in Subsection 2.1. We start by proving separatedness below.

(3.15) Proposition. The index set $\Lambda_{\alpha}^{c}$ is separated by $\omega_{\alpha}$ with

$$
C_{\Lambda_{\alpha}^{c}}=\min \left\{g, 1+c^{2}, 1+\tau^{2}, 1+\tau\right\},
$$

where $c=\inf _{\lambda \in \Lambda_{\alpha}^{c}} \gamma_{j} g^{j(1-\alpha)}$.

Proof. Let $\lambda, \lambda^{\prime} \in \Lambda, \lambda \neq \lambda^{\prime}$. If $j \neq j^{\prime}$, then

$$
\omega\left(\lambda, \lambda^{\prime}\right)=g^{\left|j-j^{\prime}\right|}\left(1+d\left(\lambda, \lambda^{\prime}\right) \geq g .\right.
$$

Thus we can suppose $j=j^{\prime}$, so that $\omega\left(\lambda, \lambda^{\prime}\right)=1+d\left(\lambda, \lambda^{\prime}\right)$. Now, if $l \neq l^{\prime}$ we estimate

$$
\left|\Delta \theta_{\lambda}\right|^{2}=\left|l-l^{\prime}\right|^{2} \gamma_{j}^{2} \geq c^{2}\left|l-l^{\prime}\right|^{2} g^{-j(2-2 \alpha)} \geq c^{2} g^{-j(2-2 \alpha)},
$$

whence

$$
\omega\left(\lambda, \lambda^{\prime}\right) \geq 1+c^{2} g^{j(2-2 \alpha)} g^{-j(2-2 \alpha)}=1+c^{2} .
$$

Thus we can finally suppose $j=j^{\prime}, l=l^{\prime}$ and $k \neq k^{\prime}$. If $k_{2} \neq k_{2}^{\prime}$ we estimate

$$
\begin{aligned}
\left\|\Delta x_{\lambda}\right\|^{2} & =\left\|R_{\theta_{\lambda}}^{-1} D_{s_{\lambda}}^{-1} \tau\left(k-k^{\prime}\right)\right\|^{2} \\
& =\tau^{2}\left\|D_{s_{\lambda}}^{-1}\left(k-k^{\prime}\right)\right\|^{2} \\
& =\tau^{2}\left[g^{-j 2}\left(k_{1}-k_{1}^{\prime}\right)^{2}+g^{-j 2 \alpha}\left(k_{2}-k_{2}^{\prime}\right)^{2}\right] \\
& \geq \tau^{2} g^{-j 2 \alpha}\left(k_{2}-k_{2}^{\prime}\right)^{2} \\
& \geq \tau^{2} g^{-j 2 \alpha}
\end{aligned}
$$

whence

$$
\omega\left(\lambda, \lambda^{\prime}\right) \geq 1+\tau^{2} g^{j 2 \alpha} g^{-j 2 \alpha}=1+\tau^{2} .
$$

Otherwise, if $k_{2}=k_{2}^{\prime}$ and $k_{1} \neq k_{1}^{\prime}$ we estimate

$$
\left|\left\langle\Delta x_{\lambda}, e_{\theta_{\lambda}}\right\rangle\right|=\left|g^{-j} \tau\left(k_{1}-k_{1}^{\prime}\right)\right|=g^{-j} \tau\left|k_{1}-k_{1}^{\prime}\right| \geq g^{-j} \tau,
$$

whence

$$
\omega\left(\lambda, \lambda^{\prime}\right) \geq 1+g^{j} g^{-j} \tau=1+\tau .
$$

Next we examine the admissibility of $\omega_{\alpha}$ restricted to $\Lambda_{\alpha}^{c}$. This property has actually been verified in 22 and we arrive at the following result.

(3.16) Proposition. The index distance $\omega_{\alpha}$ on $\Lambda_{\alpha}^{c}$ is 2-admissible for all $\alpha \in[0,1]$.

Proof. [22, Proposition 3.6].

We can finally apply our machinery to obtain the following localization result for $\alpha$-curvelets.

(3.17) Theorem. Assume we have an $\alpha$-curvelet frame which is $(N+L)$-localized with respect to $\omega_{\alpha}$, with $N$ and $L$ satisfying (2.15), Then the dual frame is $N^{+}$-localized, with $N^{+}$given by (2.16).

Proof. Just apply Theorem (2.14) taking into account Lemma (3.13) 


\subsubsection{Shearlet-type Parametrization}

This subsection studies the admissibility and separatedness of so-called shearlet-type parametrizations as introduced in 22]. This paramatrization discretizes the directional parameter uniformly in slope rather than in angle, which is advantageous for digital implementations. This is done by means of the shear transformation

$$
S_{t}:=\left(\begin{array}{cc}
1 & t \\
0 & 1
\end{array}\right)
$$

which replaces the usual rotation $R_{\theta}$ with $t=\tan \theta$.

(3.19) Definition. Let $\alpha \in[0,1], g>1$ and $\tau>0$. Further, let $\left(\eta_{j}\right)_{j \in \mathbb{Z}}$ and $\left(L_{j}\right)_{j \in \mathbb{Z}}$ be sequences of positive real numbers with $\eta_{j} \asymp g^{-j(1-\alpha)}$ and $L_{j} \lesssim g^{j(1-\alpha)}$. An $\alpha$-shearlet parametrization is given by an index set of the form

$$
\Lambda_{\alpha}^{s}:=\left\{(j, l, k) \in \mathbb{Z} \times \mathbb{Z} \times \mathbb{Z}^{2}:|l| \leq L_{j}\right\}
$$

and a mapping

$$
\Phi^{s}(\lambda):=\left(s_{\lambda}, \theta_{\lambda}, x_{\lambda}\right):=\left(g^{j}, \arctan \left(l \eta_{j}\right), S_{\tan \theta_{\lambda}}^{-1} D_{s_{\lambda}}^{-1} \tau k\right) .
$$

(3.20) Remark. We may suppose to work with a scaling function and thus consider only positive scales $j \in$ $\mathbb{N}$, but this would require additional notation and inessential slight complications on the paramatrization. Anyway, all the arguments go through with or without scaling functions.

Similarly to the $\alpha$-curvelet case, we first prove that $\omega_{\alpha}$ separates $\Lambda_{\alpha}^{s}$.

(3.21) Proposition. The index set $\Lambda_{\alpha}^{s}$ is separated by $\omega_{\alpha}$, with

$$
C_{\Lambda_{\alpha}^{s}}=\min \left\{g, 1+c^{2}\left(1+C^{2}\right)^{-2}, 1+\tau^{2}, 1+\tau\left(1+C^{2}\right)^{-1 / 2}\right\},
$$

where $c=\inf _{\lambda \in \Lambda_{\alpha}^{s}} \eta_{j} g^{j(1-\alpha)}$ and $C=\sup _{j \in \mathbb{Z}} L_{j} \eta_{j}$.

Proof. Let $\lambda, \lambda^{\prime} \in \Lambda, \lambda \neq \lambda^{\prime}$. If $j \neq j^{\prime}$, then

$$
\omega\left(\lambda, \lambda^{\prime}\right)=g^{\left|j-j^{\prime}\right|}\left(1+d\left(\lambda, \lambda^{\prime}\right) \geq g .\right.
$$

Thus we can suppose $j=j^{\prime}$, so that $\omega\left(\lambda, \lambda^{\prime}\right)=1+d\left(\lambda, \lambda^{\prime}\right)$. If $l \neq l^{\prime}$ we estimate $\left|\Delta \theta_{\lambda}\right|^{2}$. By the mean value theorem we have

$$
\left|\Delta \theta_{\lambda}\right|^{2}=\left|\arctan \left(l \eta_{j}\right)-\arctan \left(l^{\prime} \eta_{j}\right)\right|^{2}=\eta_{j}^{2}\left|l-l^{\prime}\right|^{2}\left(\frac{1}{1+\xi^{2}}\right)^{2}
$$

for some $\xi,|\xi| \leq\left|\max \left(l, l^{\prime}\right)\right| \eta_{j} \leq L_{j} \eta_{j} \leq C$, so that

$$
\left|\Delta \theta_{\lambda}\right|^{2} \geq c^{2} g^{-j(2-2 \alpha)}\left|l-l^{\prime}\right|^{2}\left(1+C^{2}\right)^{-2} \geq c^{2} g^{-j(2-2 \alpha)}\left(1+C^{2}\right)^{-2},
$$

whence

$$
\omega\left(\lambda, \lambda^{\prime}\right) \geq 1+c^{2} g^{j(2-2 \alpha)} g^{-j(2-2 \alpha)}\left(1+C^{2}\right)^{-2}=1+c^{2}\left(1+C^{2}\right)^{-2} .
$$

Thus we can finally suppose $j=j^{\prime}, l=l^{\prime}$ and $k \neq k^{\prime}$. If $k_{2} \neq k_{2}^{\prime}$ we estimate

$$
\begin{aligned}
\left\|\Delta x_{\lambda}\right\|^{2} & =\left\|S_{l \eta_{j}}^{-1} D_{g^{j}}^{-1} \tau\left(k-k^{\prime}\right)\right\|^{2} \\
& =\tau^{2}\left\{\left[g^{-j}\left(k_{1}-k_{1}^{\prime}\right)-l \eta_{j} g^{-j \alpha}\left(k_{2}-k_{2}^{\prime}\right)\right]^{2}+g^{-j 2 \alpha}\left(k_{2}-k_{2}^{\prime}\right)^{2}\right\} \\
& \geq \tau^{2} g^{-j 2 \alpha}\left(k_{2}-k_{2}^{\prime}\right)^{2} \\
& \geq \tau^{2} g^{-j 2 \alpha}
\end{aligned}
$$

whence

$$
\omega\left(\lambda, \lambda^{\prime}\right) \geq 1+\tau^{2} g^{j 2 \alpha} g^{-j 2 \alpha}=1+\tau^{2} .
$$


Otherwise, if $k_{2}=k_{2}^{\prime}$ and $k_{1} \neq k_{1}^{\prime}$ we estimate

$$
\begin{aligned}
\left|\left\langle\Delta x_{\lambda}, e_{\theta_{\lambda}}\right\rangle\right| & =\left|g^{-j} \tau\left(k_{1}-k_{1}^{\prime}\right) \cos \theta_{\lambda}\right| \\
& =g^{-j} \tau\left|k_{1}-k_{1}^{\prime}\right| \cos \theta_{\lambda} \\
& =g^{-j} \tau\left|k_{1}-k_{1}^{\prime}\right| \cos \arctan \left(l \eta_{j}\right) \\
& =g^{-j} \tau\left|k_{1}-k_{1}^{\prime}\right| \frac{1}{\sqrt{1+l^{2} \eta_{j}^{2}}} \\
& \geq g^{-j} \tau\left(1+C^{2}\right)^{-1 / 2}
\end{aligned}
$$

whence

$$
\omega\left(\lambda, \lambda^{\prime}\right) \geq 1+g^{j} g^{-j} \tau\left(1+C^{2}\right)^{-1 / 2}=1+\tau\left(1+C^{2}\right)^{-1 / 2} .
$$

Finally, it only remains to see the matter of admissibility. As before, we refer to [22].

(3.22) Proposition. The index distance $\omega_{\alpha}$ on $\Lambda_{\alpha}^{s}$ is 2-admissible for all $\alpha \in[0,1]$.

Proof. [22, Proposition 3.6].

We conclude by stating the corresponding localization result for $\alpha$-shearlet frames.

(3.23) Theorem. Assume we have an $\alpha$-shearlet frame which is $(N+L)$-localized with respect to $\omega_{\alpha}$, with $N$ and $L$ satisfying (2.15), Then the dual frame is $N^{+}$-localized, with $N^{+}$given by (2.16).

Proof. Just apply Theorem (2.14) taking into account Lemma (3.13)

\section{References}

[1] A. Aldroubi, A. Baskakov, and I. Krishtal. Slanted matrices, banach frames, and sampling. Journal of Functional Analysis, 255(7):1667-1691, 2008.

[2] R. Balan. The noncommutative Wiener lemma, linear independence, and spectral properties of the algebra of time-frequency shift operators. Transactions of the American Mathematical Society, 360(7):3921-3942, 2008.

[3] R. Balan, P.G. Casazza, C. Heil, and Z. Landau. Density, overcompleteness, and localization of frames. I. Theory. Journal of Fourier Analysis and Applications, 12(2):105-143, 2006.

[4] R. Balan, P.G. Casazza, C. Heil, and Z. Landau. Density, overcompleteness, and localization of frames. II. Gabor systems. Journal of Fourier Analysis and Applications, 12(3):307-344, 2006.

[5] A.G. Baskakov. Asymptotic estimates for elements of matrices of inverse operators, and harmonic analysis. Sibirskii Matematicheskii Zhurnal, 38(1):14-28, 1997.

[6] A.G. Baskakov. Estimates for the entries of inverse matrices and the spectral analysis of linear operators. Izvestiya: Mathematics, 61:1113, 1997.

[7] A.G. Baskakov and I.A. Krishtal. Memory estimation of inverse operators. Arxiv preprint arXiv:1103.2748, 2011.

[8] L. Borup and M. Nielsen. Frame decompositions of decomposition spaces. Journal of Fourier Analysis and Applications, 13:39-70, 2007.

[9] E. Candès. Ridgelets: Theory and applications, 1998. PhD thesis, Stanford University.

[10] E.J. Candès and D.L. Donoho. New tight frames of curvelets and optimal representations of objects with piecewise $C^{2}$ singularities. Communications on Pure and Applied Mathematics, 57(2):219-266, 2004.

[11] O. Christensen. An Introduction to Frames and Riesz Bases. Birkhäuser, 2003.

[12] E. Cordero and K. Gröchenig. Localization of frames II. Applied and Computational Harmonic Analysis, 17(1):29-47, 2004. 
[13] S. Demko, W.F. Moss, and P.W. Smith. Decay rates for inverses of band matrices. Mathematics of Computation, 43(168):491-499, 1984.

[14] Candès E.J. and L. Demanet. The curvelet representation of wave propagators is optimally sparse. Communications on Pure and Applied Mathematics, 58:1472-1528, 2004.

[15] M. Fornasier and K. Gröchenig. Intrinsic localization of frames. Constructive Approximation, $22(3): 395-415,2005$.

[16] M. Frazier, B. Jawerth, and G. Weiss. Littlewood-Paley theory and the study of function spaces. Number 79. American Mathematical Soc., 1991.

[17] F. Futamura. Localizable operators and the construction of localized frames. Proceedings of the American Mathematical Society, 137(12):4187-4197, 2009.

[18] K. Gröchenig. Localization of frames, Banach frames, and the invertibility of the frame operator. Journal of Fourier Analysis and Applications, 10(2):105-132, 2004.

[19] P. Grohs. Ridgelet-type frame decompositions for Sobolev spaces related to linear transport. J. Fourier Anal. Appl., 18(2):309-325, 2012.

[20] P. Grohs. Bandlimited shearlet frames with nice duals. Journal of Computational and Applied Mathematics, 243:139-151, 2013.

[21] P. Grohs. Intrinsic localization of anisotropic frames. Applied and Computational Harmonic Analysis, $35: 264-283,2013$.

[22] P. Grohs, S. Keiper, G. Kutyniok, and M. Schaefer. $\alpha$-molecules: Wavelets, curvelets, shearlets, ridgelts and beyond. In Proceedings SPIE 2013, 2013.

[23] P. Grohs and G. Kutyniok. Parabolic molecules. Foundations of Computational Mathematics, 2014. in press.

[24] K. Gröchenig and S. Samarah. Nonlinear approximation with local fourier bases. Constructive Approximation, 16(3):317-331, 2000.

[25] J. Gustavsson. On interpolation of weighted $L^{p}$-spaces and Ovchinnikov's theorem. Studia Mathematica, 72(3):237-251, 1982.

[26] S. Jaffard. Propriétés des matrices "bien localisées" pres de leur diagonale et quelques applications. Annales de l'Institut Henri Poincaré (C) Analyse Non Linéaire, 7(5):461-476, 1990.

[27] S. Keiper. A flexible shearlet transform - sparse approximation and dictionary learning, 2013. Bachelor's thesis, TU Berlin.

[28] I.A. Krishtal. Wiener's lemma and memory localization. Journal of Fourier Analysis and Applications, 17(4):674-690, 2011.

[29] D. Labate, W.-Q. Lim, G. Kutyniok, and G. Weiss. Sparse multidimensional representation using shearlets. In SPIE Proc. 5914, SPIE, Bellingham, WA, pages 254-262, 2005.

[30] Q. Sun. Wiener's lemma for infinite matrices. Transactions of the American Mathematical Society, 359(7):3099-3124, 2007.

[31] Q. Sun. Wiener's lemma for infinite matrices II. Constructive Approximation, 34(2):209-235, 2011.

\section{A Auxiliary Results}

(A.1) Lemma. For all $t, t^{\prime}, t^{\prime \prime} \in \mathbb{R}$ and $\beta \leq 2$,

$$
\left|t-t^{\prime}\right|+\beta \min \left(t, t^{\prime}\right) \leq\left|t-t^{\prime \prime}\right|+\left|t^{\prime \prime}-t^{\prime}\right|+\beta \min \left(t, t^{\prime}, t^{\prime \prime}\right) ;
$$

if $\beta \in[0,2]$, then

$$
\left|t-t^{\prime}\right|+\beta \min \left(t, t^{\prime}\right) \leq\left|t-t^{\prime \prime}\right|+\left|t^{\prime \prime}-t^{\prime}\right|+\beta\left\{\begin{array}{l}
\min \left(t, t^{\prime \prime}\right) \\
\min \left(t^{\prime \prime}, t^{\prime}\right)
\end{array} .\right.
$$


Proof. If $t^{\prime \prime} \geq \min \left(t, t^{\prime}\right)$, then $\min \left(t, t^{\prime}\right)=\min \left(t, t^{\prime}, t^{\prime \prime}\right)$, hence $\beta \min \left(t, t^{\prime}\right)=\beta \min \left(t, t^{\prime}, t^{\prime \prime}\right)$ for any $\beta \in \mathbb{R}$. Thus the first inequality of the thesis reduces to the usual triangle inequality. But if $t^{\prime \prime} \leq \min \left(t, t^{\prime}\right)$, then $\min \left(t, t^{\prime}\right) \geq \min \left(t, t^{\prime}, t^{\prime \prime}\right)$, and we need the triangle inequality to counterbalance this effect. Of course we can suppose $t^{\prime \prime} \leq t \leq t^{\prime}$, so that $\min \left(t, t^{\prime}\right)=t$ and $\min \left(t, t^{\prime}, t^{\prime \prime}\right)=t^{\prime \prime}$. The left term is now $t^{\prime}+(\beta-1) t$, while the right term is $t^{\prime}+t+(\beta-2) t^{\prime \prime}$, so that the inequality is equivalent to $(\beta-2) t \leq(\beta-2) t^{\prime \prime}$, which is true for $t^{\prime \prime} \leq t$ if $\beta \leq 2$.

If in addition $\beta \geq 0$, we have $\beta \min \left(t, t^{\prime}, t^{\prime \prime}\right) \leq \beta m$, where $m$ is either $\min \left(t, t^{\prime \prime}\right)$ or $\min \left(t^{\prime \prime}, t^{\prime}\right)$, whence then second couple of inequalities.

(A.2) Lemma. For all $t, t^{\prime}, t^{\prime \prime} \in \mathbb{R}$

$$
\max \left(\min \left(t, t^{\prime \prime}\right), \min \left(t^{\prime \prime}, t^{\prime}\right)\right) \leq \min \left(\max \left(t, t^{\prime \prime}\right), \max \left(t^{\prime \prime}, t^{\prime}\right)\right) .
$$

Proof. We can suppose $t \leq t^{\prime}$ and check the left term $L$ and the right term $R$ in each case.

$t^{\prime \prime} \leq t \leq t^{\prime}: \quad L=t^{\prime \prime}, R=t$.

$t \leq t^{\prime \prime} \leq t^{\prime}: \quad L=t^{\prime \prime}, R=t^{\prime \prime}$.

$t \leq t^{\prime} \leq t^{\prime \prime}: \quad L=t^{\prime}, R=t^{\prime \prime}$. 